\title{
Business Finder - A Tool for Regional Networking among Organizations
}

\author{
Tim Reichling, Benjamin Moos, and Volker Wulf \\ Institute of Information Systems, University of Siegen, Germany, \{tim.reichling, benjamin.moos, \\ volker.wulf\}@uni-siegen.de
}

\begin{abstract}
Networks of organizations improve the competitiveness of its member companies. Computer applications can make the competencies of organizations more visible to encourage companies to find matching costumers, suppliers, or cooperation partners. Business Finder (BF) is a tool for improving mutual awareness among small and medium enterprises (SME) in regional networks. BF is based on text matching algorithms already applied among human actors within the field of knowledge management. Being integrated into the ordinary document management, BF allows creating updated, comprehensive, and detailed profiles of an organization's competencies and activities. Searching on profiles of other organizations enables identifying potential partners. The design of BF is based on an empirical study into networking needs among SME in the IT domain. The prestudy together with an early evaluation study was conducted in the German region of Siegen-Wittgenstein.
\end{abstract}

\section{Introduction}

Networks of geographically collocated companies are widely known to offer its members competitive advantages. Those advantages may be found in lower transportation costs, better knowledge spill-overs, more intense cooperation along the supply chain, or faster innovation cycles. According to Porter (1998), networks of highly interconnected and geographically proximate companies along a value chain, so called clusters, strongly encourage regional economy and create competitive advantage to its constituting companies. Popular examples to underpin this thesis are Silicon Valley for IT, Hollywood for movie production or the Ruhr Area in Germany for metal-work.

In this paper we focus on the role of IT to support transparency and mutual awareness in regional networks of companies. We do not focus on clusters exclusively since we also consider regional networks of companies which do not (yet) show the distinct properties of clusters. Creating and supporting networks of com- 
panies is often taken to be a central goal in regional economic intervention. However, technical approaches to support networks are rare.

With regard to the domain of knowledge management, expertise recommender systems have proved to be promising technologies to support networks of human actors within organizations (cf. Hinds and Pfeffer 2003, Huysman and Wulf 2006, Reichling et al. 2005, 2007). In this paper, we investigate whether these technologies can be successfully applied to support awareness and transparency in networks of regional companies.

The argument is structured as follows. First, we analysed requirements of SME with regard to mutual awareness. The study was conducted with the business development department of the Siegen-Wittgenstein region (Germany). Based on these findings, we designed the Business Finder, a keyword based search engine for regional SME. Business Finder is a synthesis of two existing technologies: an expertise recommender system designed for encouraging expertise sharing in large organizations (Reichling et al. 2007) and the database of regional companies (DRC) that is hosted by the business development department. DRC is a web based directory containing basic information about regional SME from media and IT sectors in the broadest sense. A first evaluation study focuses on the systems performance (compared to existing search engines like Google) and the actual value that it provides to potential users.

\section{Related work}

A variety of studies indicate that structures of regional proximity and interconnectedness to be important success factors for companies. While globalization and new media appear to outperform these seemingly antiquated assets, they seem to maintain their significance. Porter (1998) labels this phenomenon The Location Paradox. Lower costs for transportation or resources can not sufficiently explain why companies of a given sector appear to settle down in the same region.

Donhauser (2006) finds incentives for regional concentration in an increasing potential for innovation, productivity and growth by better preconditions for cooperation. These are a result of different circumstances: First, regional proximity leads to vivid informal communication among human actors even across companies resulting in a rapid diffusion of expertise and best practices. Second, the regional proximity often goes along with the creation of a highly specialized "Labour Pool" (Schiele 2003) from which regional companies can select their staff while saving time and costs for training. Third, another issue concerned with knowledge dissemination among actors of interconnected enterprises is trust (Porter 1998). Since trust is a property of social ties, social capital (SC) (cf. Bourdieu 1983) affects processes of knowledge dissemination (Huysman and Wulf 2004). Following Wolfe (2002) social capital is not transferable among human actors. It 
encourages the actors' (across companies) willingness to mutually support each other, initiate business cooperation and share knowledge.

Besides the aspect of an improved information exchange, clusters are also characterized by stronger competition as a result of a large number of enterprises in the same sector and improved transparency. According to Porter (1998) „Companies can mitigate many input-cost disadvantages through global sourcing, rendering the old notion of comparative advantage less relevant. Instead, competitive advantage rests on making more productive use of inputs, which requires continual innovation. [...] Without vigorous competition a cluster will fail". Porter realizes that cooperation and competition can coexist within a cluster and do not exclude each other. In fact both are required for successful clusters.

We now turn to the question of how IT can contribute to the development of regional networks which may lead to the formation of cluster structures. Krätke and Scheuplein (2001) suggest that IT systems could support cluster recognition and analysis to enable business development departments to better ground decisions on political interventions. They state that visualizing cluster boundaries as well as internal interconnections and offering comparisons of regional and super regional clusters are central requirements for this purpose.

IT systems that perform algorithmic matching of model based descriptions, recommender systems according to Resnick and Varian (1997), gain importance in different domains of information and communication technology (ICT) (cf. Balabanovic and Shoham 1997, Resnick 1994), especially knowledge management (cf. Hinds and Pfeffer 2003, Huysman and Wulf 2004, Reichling et al. 2005, 2007). With respect to cluster support, Porter (1998) suggests that IT systems could create awareness on other actors within a cluster. Similarly, Leuninger and Held (2003) - without specifying certain technological approaches - argue for an IS based address and communication platform.

Up to now, IT had little significance in dedicated cluster support and is treated neglectfully in the literature. Instead, existing technologies from other domains appear promising for network development among geographical proximate companies. As such, we can find common search engines like Google or Yahoo which cover websites in general. Their results can hardly be limited to regional companies. We also find directories of regional companies, hosted and maintained by local business development units that often suffer from out-dated and incomplete profiles or high efforts to keep them updated. We can further consider ontologybased approaches (Blomquist 2007). Omitting imprecise or ambiguous results, ontologies offer sophisticated search functionality for companies' competencies. However, meaningful ontologies need to be created and updated, which means a considerable effort. Hence, this kind of technology so far has not been applied for cluster support. Another aspect that can be found at many clusters is the existence of a common website representing the cluster as such (Sölvell et al. 2003). While it is unchallenged that representation towards the external market is highly important, it is remarkable that these sites in general do not contribute to foster connections between actors within clusters. 


\section{Business Finder: Approach and empirical study}

Based on the findings above and promising results of recommender systems in several different domains, we decided to apply recommender technology to support transparency and awareness in regional networks. The BF approach goes beyond a simple address database which most likely does not improve transparency of a company's competencies and activities sufficiently. Our study covers requirements analysis, system design and a brief evaluation of the BFs performance.

\subsection{Methods and field of application}

The district Siegen-Wittgenstein has a population of 290,000 people. Siegen, as the regional centre, denotes the most populated town and hosts most of the SME in the region. As mentioned above, with respect to the media and IT sector, Siegen-Wittgenstein can hardly be denoted as cluster according to Porter (1998) since interconnections between companies can rarely be found. To a large extent business transactions are accomplished with external partners even though potential consumers, suppliers or cooperation partners exist within the region. Furthermore, no central database exists (or is widely accepted) that covers recent profiles of regional companies. The 'closest candidate' to such a database - from our point of view - is a public database that is hosted by the regional business development department which we refer to as the database of regional companies (DRC). DRC is a yellow page (YP) like directory that contains elementary data of selected regional companies (name, address, contact information, CEO, basic competencies etc.). The data is updated occasionally by employees of the business development, but no more often that twice a year on average. Details about a company's products, services, processes or methods are missing. DRC can be requested via a web front end.

We worked together with members of the regional business development who runs the DRC. They found their system to be rarely used by regional companies. Obviously, the contents of the database were recognized as out of date, poor and (in some cases) redundant to the companies' websites. Hence, basic objectives of our study were: Concept and design of an IS to create transparency of competencies and activities as well as mutual awareness of potential transaction partners within the region which may enable network structures to grow. The IS should be embedded into the existing DRC in order to improve its search results by offering data that is more comprehensive and up to date.

Our study followed basically an action research (AR) approach (Susman and Everd, 1978, Wulf and Rohde, 1995). We applied one AR cycle consisting of the five steps of diagnosing, action planning, action taking, evaluation and specifying/learning in a slightly adjusted way. Formally, the regional business develop- 
ment department can be referred to as our client. For the steps of analysing and evaluation, we relied on qualitative and ethnographical methods (Flick 2002) and conducted semi-structured and open-ended interviews with participants from representative companies (one participant per company). For the evaluation study further participants were asked to judge the results returned by the BF system.

For the requirements analysis (diagnosing) we conducted 16 semi-structured and open-ended interviews with participants of SME from different sectors: software development, web design / advertisement, trade, storage, hardware construction and training. Additionally, we had two participants from large scale enterprises. These and the participant from the training company were supposed to be 'consumers' of IT while the others were supposed to be 'suppliers'. In case of the IT suppliers, which were mainly rather small enterprises, most of the participants were founders and directors of their own companies. The participants of the two large enterprises were an IT manager of a regional brewery and a director of quality management of a switchboard manufacturer, respectively. In a later stage of the interview, interviewees were given a brief outline of our concept of network support by a dedicated search engine. The interviews were recorded by a tape recorder with the participants' agreement.

In a further step, we analyzed the interviews and generated requirements for the BF system. From that, we developed the basic concept of the BF, a central database containing rich keyword-based profiles of regional companies that can be requested via a web front-end (action planning). We implemented BF following the requirements and integrated it into the DRC system (action taking). To evaluate the BF system, we first compared its results to those of existing search engines Google, Google Maps and the former DRC. As part of another interview session (evaluation), we presented $\mathrm{BF}$ to four potential users from regional companies of the media and IT sector. We finally interpreted and summarized the results (specifying / learning). The subsequent sections will describe the essential results of the steps 2, 3 and 4 in more detail.

\subsection{Requirement for cluster support}

While most of the business transactions, as mentioned above, are accomplished with partners from outside the region, the participants expressed their general willingness to transact with regional partners. However, especially for suppliers of immaterial, digital products (web designers or software developers who constitute a considerable part of our participants) it is easy to transfer their 'goods' over far distances, as interviewees stated. Hence, unlike other industry sectors, geographical proximity is no necessary precondition for cooperation. Besides this obvious argument concerned with the IT sector, other (more subtle) reasons for super regional cooperation were discussed. The companies were "too small and insignificant" to carry out large scale orders, as one participant stated. Similarly, another 
participant judged that regional companies were not likely to offer large scale orders which were the only orders they could profitably accept.

With regard to the selecting cooperation partners, participants stated that mutual trust between representatives was the main precondition for successful cooperation. In order to maintain the level of customer satisfaction, the actors value the cooperation partners' high quality demands that fit to their own demands. When former unknown partners shall be assessed, the actors rely heavily on oral recommendations given by trusted partners. As one interviewee states: "See, it works just about personal contacts or word-of-mouth recommendation, based on trust".

We further asked the interviewees for their attitude on existing databases, search engines and yellow page directories. We found that all the companies that were covered by our inquiry had registered to numerous YP directories. However, from their experience participants were very sceptical about these this kind of systems: YP directories actually had little value measured against the effort of keeping the companies profiles up to date. At the same time the effort increases with the number of systems that companies were subscribed in. Merely two of the interviewees reported successful business transactions as a result of the system subscriptions. Hence, companies limit their efforts to a small number of well chosen systems.

Essential criteria of choosing YP directories that were given by participants are quality and up-to-dateness. Furthermore, the participants' impression of the operators in terms of maintenance and care is an important criterion. Again, trust towards the directories' operators appears to be a central factor. As one participant stated, he had lost trust in one directory after he discovered “...massive spelling mistakes in name or address $[\ldots]$ such that you get the impression $[\ldots]$ they try to make you update your profile".

Controversy perspectives showed up concerned with the directories' focus. While some participants found highly specialized and sector specific directories useful, others demanded for comprehensiveness. In both cases completeness in terms of the covered companies and a critical mass of registered companies is required. At the same time, the actors worry about potential masses of advertisement (spam) that might go along with a large number of subscribers. Surprisingly with regard to their attitude towards existing databases (see above), all the interviewees expressed their interest and willingness to contribute to the BF after launching the first prototype.

The statements above illustrate that up-to-dateness and effort spent on maintaining it are central concerns to the participants. Hence, in order to minimize this effort, BF should be capable of automatically create and update profiles from existing data. This data, as interviewees stated, may consist of specific text documents related to the company like product specifications, advertisement, flyers, websites or newsletters. While some companies websites are updated rarely (one participant reported once in three years), newsletters - by definition - provide highly recent information about products, offers or services. Since many compa- 
nies periodically send out newsletters, these appear to be a promising source of relevant and recent information about the company.

With regard to indicating sources of data, privacy concerns apparently had to be discussed as well. From the interviews we learned that product specifications, flyers, advertising material or newsletters did not collide with privacy concerns, since these are public by definition. On the other hand, internal documents which may contain highly relevant information should not be accessed, even though only basic information would be stored (see below). Some participants stated that careful selection of relevant (locally stored) documents with respect to privacy concerns was no significant reduction of effort but rather a "shift of effort".

\subsection{Business Finder: concept and implementation}

From the results of the empirical study we derived a set of requirements for the design of the BF system. BF, as a recommender system for regional enterprises of the media and IT sector, should provide recent and comprehensive profiles of companies while the effort for creating and maintaining them should be minimized by utilizing existing sources of data that do not collide with the users privacy concerns. For instance, documents like product descriptions, advertising material, newsletters etc. are adequate resources.

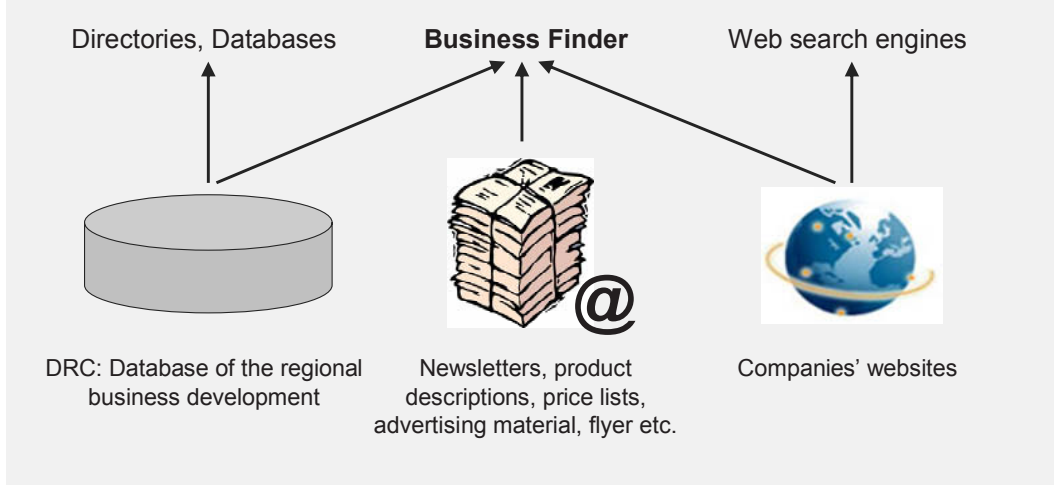

Figure 1. Basic concept of BF including three sources of data

Based on these requirements, we designed the BF system to meet the reqirements of the SME in the region of Siegen-Wittgenstein. BF can be regarded as a central database which stores keyword-based enterprise profiles and matches them against requests. Profiles consist of two types of data. First, keyword profiles (cf. Reichling et al. 2007) are an aggregate of the contents of arbitrary company specific documents and websites (see above). Second, elementary data is accessed which we obtain from the DRC. Hence, BF covers information from three sources of 
data: The DRC which is a traditional YP directory, Websites, which web search engines like Google or Yahoo use and arbitrary text documents chosen by responsible actors in the company. The latter two are regarded as analogue by their nature, so both are aggregated within the keyword profile. The concept of BF is shown in figure 1.

The implementation of BF is based on the ExpertFinding (EF) system (Reichling et al. 2007), an expertise recommender system for large or distributed enterprises. Its main purpose is creating awareness of activities, expertises and experiences of actors within an organization in order to foster transparency and collaboration of the staff. While EF is essentially knowledge management technology, from its purpose it is very similar to BF. The EF client system provides a software tool which allows users to easily select documents or folders from the local file system, specifying path, age, author and file format (typically doc, pdf, ppt, etc.). From these specifications EF creates keyword profiles which are an aggregate of the textual contents of all specified documents (cf. Reichling et al. 2007). For privacy concerns, all this takes place locally on the client machine. Users are given the opportunity to inspect and eventually correct their keyword profile before uploading it to the server.

As EF provides basic technology that can be used for BF as well, we decided to ground the BF on EF technology. Meanwhile, BF differs from EF in some elementary points: First, BF contains companies' profiles instead of user profiles (which is merely irrelevant from a technical point of view). Second, since arbitrary text documents, newsletters and websites are included, documents reach the server via manifold ways. 1) Via the web-based front-end (see figure 2a) users can select and upload single documents ${ }^{1}$ in order to create the keyword profile. As in EF, keyword profiles can be edited in order to remove single keywords. 2) In order to receive newsletters, we set up an email account which the letter can be sent to - so mailing lists need to be extended with that address in order to automatically include the newsletter into the keyword profile. 3) The web front-end further provides facilities to enter the companies' website's URL (see figure 2a). A dedicated server side component of BF periodically scans all the registered websites, just as web search engines do. The BF system was installed on a machine at the regional business development. It has direct access to the DRC database in order to access its elementary data.

Figure $2 \mathrm{~b}$ shows the searching facility of BFs web front-end. The way it works is analogous to web search engines and needs no further explanation. Exemplary, figure $2 \mathrm{~b}$ shows the search results for the search term Werbung (advertisement). Multiple keywords can be entered as well. The semitransparent tooltip in figure $2 b$ shows further details about the search results. Since rich data of the companies is stored, tooltips appeared to be a suitable way of visualizing.

\footnotetext{
${ }^{1}$ The BF prototype allows for uploading single documents. Eventual later version should support upload of entire folders as well
} 


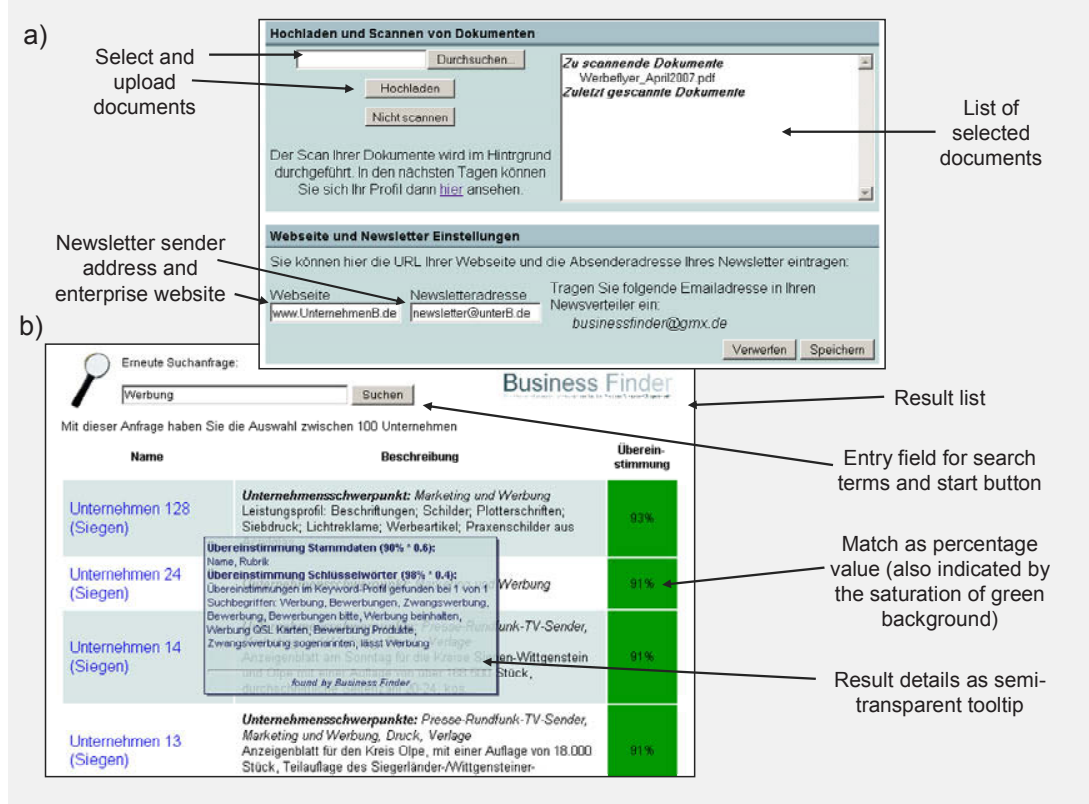

Figure 2. a) Uploading documents and specifying URL of the companies' websites and b) Result list of matching companies

\subsection{Evaluation of the Business Finder}

In March 2007, BF was presented to representatives of regional economy and launched shortly afterwards. We evaluated the design of BF on two levels. First, we presented the application to four different companies. Two of them were regional small-sized IT companies which offered software products and services. The other two companies, a large brewery and a producer of switching cabinets, both large scale enterprises which demand IT services on the regional market. In a second phase, we did a comparative analysis of search results created by BF and those of Google, Google Maps and two regional databases for IT companies ${ }^{2}$. It should be mentioned that at the time of the evaluation elementary data from the former DRC and websites were the only input to BF to create profiles from. No further documents like advertising materials or newsletters were used. Hence, the participating companies did not have any efforts to create their profiles (by means of selecting appropriate documents).

\footnotetext{
${ }^{2}$ The first database was given by DRC, the second database was the search engine hosted by the regional department of the Chamber of Industry and Commerce (Industrie- und Handelskammer, IHK)
} 
When presenting the system to the regional companies, the participants could try out the system and evaluate the search results. We also asked the participants for potential improvements. While all of the participants tested BF's search engine, the participants from the IT service providers also tested the profile creation facilities. The overall judgement of the participants with respect to usability and profile creation was positive. Concerns, however, were expressed with regard to the danger of manipulation by competing companies. The participants of the two IT companies feared that competitors could manipulate the keyword profiles by uploading large amounts of documents in order to appear in the result lists more frequently and in higher positions.

To evaluate $\mathrm{BF}$ in comparison with results from other search engines, i.e. Google and the local database of IT companies, we looked for six terms that we judged to be relevant within the IT industry; Internet, database, PHP, Server, programming, Java. We chose Google as representative search engine that is widely said to return satisfactory results. Since Google is not geographically restricted we added the region's name "Siegen" as an additional search term. We chose the local database of IT companies as another point of reference in order to assess potential advantages of BF. With regard to each term of the search queries, BF outperformed both the local database and Google. As expected, Google delivered many results which were not hosted by companies.

Participants expressed some scepticism with regard to the choice of search terms. They found them too unspecific. Therefore, they were asked to enter arbitrary keywords they found relevant to find a specific local IT company - the IT companies' participants aimed at finding their own companies via BF and entered related keywords. In each case, this company was among the top five BF results. Google and the local database of IT companies performed worse. Looking for his own company, one participant of an IT company expressed satisfaction with BF's results, especially when considering that they did not have to input or update his profile: “... because you see, I was found even though I did not maintain any profile" (see above).

In a second evaluation phase, we carried out a comparative search analysis with regard to the findings recall and precision. We compared the search findings of Google, Google Maps and two local databases for IT companies using 10 IT relevant search terms. Again, we added the region's name as a search term in the cases of Google and Google-Maps. We took the 20 topmost results as representative for the search engines' results. Recall denotes the ratio of relevant results to the overall number of (potential) relevant results. Precision is defined as the ratio between the relevant results and the number of results. A result was judged to be of "relevance" in case the company was located in the Siegen-Wittgenstein region and fits the search term(s). Figure 3 gives an impression of the BF performance compared to the other four search engines. 
a)

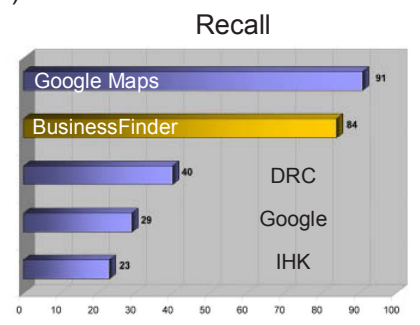

b)

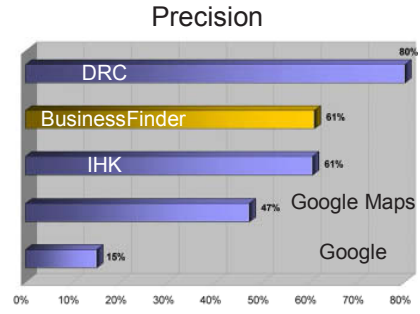

Figure 3. a) Recall and b) Precision of the search engines

With regard to both dimensions - recall and precision - BF is doing well, even though in each dimension it was outperformed by one competitor. While Google Maps appears to have the best recall (figure 3a) it does not perform well with regard to precision (figure $3 \mathrm{~b}$ ). Only $47 \%$ of its results were actually regional companies. One of the regional databases (DRC) does best with regard to precision, which is not too surprising since all of its entries are by definition from the Siegen-Wittgenstein region. However, it does not perform well with regard to recall since there are quite some companies which are not registered in the database. Combining the results for recall and precision, BF performs best.

The results of the evaluation further confirm the findings of the prestudy. The interviewees' already hinted to the fact that the existing databases of regional IT companies do not cover a sufficient amount of subscribed companies and hence do not offer a critical mass. Even if companies are registered, they may not be found due to the poor profiles kept in the database.

\section{Conclusions}

Our study in the IT sector of the Siegen-Wittgenstein region indicates that awareness among companies can foster regional networks. To offer awareness, BF created a company's profile from public, semi-public and private documents and data. An early evaluation shows pretty good precision and recall measured compared to existing web search engines (Google, Google Maps) and local databases (DRC and IHK). Given that the input to BF was restricted in this study to the companies' websites and DRC information, this is a quite remarkable result.

Future work will have to investigate into the use of BF in practise and its impact on interconnectedness and cooperation among regional companies. Our study indicates that cooperation is often hampered due to presumptions on regional companies' potential contract volume. BF may correct some of these presumptions. 


\section{References}

Ackerman, M.; Pipek, V.; Wulf, V. (Hrsg.) (2003): Preface to Sharing Expertise: Beyond Knowledge Management, MIT-Press, Cambridge, MA.

Balabanovic, M, Shoham, Y. (1997): Fab: Content-Based, Collaborative Recommendation, in Communications of the ACM, vol. 40, no. 3, 1997, 66-72.

Blomqvist E. (2007): OntoCase - A Pattern-based Ontology Construction Approach. In: Proc. of OTM 2007: ODBASE - The 6th International Conference on Ontologies, November 25-30

Bourdieu, P (1983): Ökonomisches Kapital, kulturelles Kapital, soziales Kapital, in Kreckel, Reinhard (Hg.): Soziale Ungleichheiten, Opladen 1983 (Soziale Welt, Sonderband 2) S. 183198.

Donhauser, S. (2006): Aktivierung von Wachstumspotenzialen durch Netzwerke Clusterbildung in Baden-Württemberg. In: Statistisches Monatsheft Baden-Württemberg 4, S. 18-23.

Flick, U. (2002): Qualitative Sozialforschung : Eine Einführung, Rowohlt Taschenbuch Verlag, Hamburg.

Hinds, P. J. and Pfeffer, J. (2003): Why Organizations Don't "Know What They Know", in Sharing Expertise - Beyond Knowledge Management, MIT Press, Cambridge, 2003, pp. 3-26.

Huysman M., Wulf, V. (2004): Social Capital and Information Technology. MIT-Press, Cambridge, MA 2004.

Huysman, M.; Wulf, V. (2006): IT to Support Knowledge Sharing in Communities: Towards a Social Capital Analysis, Journal of Information Technology (JIT), Vol. 21, No. 1, pp. 40-51

Krätke, S.; Scheuplein, C. (2001): Produktionscluster in Ostdeutschland: Methoden der Identifizierung und Analyse -Kurzfassung- Eine Studie im Auftrag der Otto Brenner Stiftung. Berlin.

Leuninger, S; Held, H (2003): Kommunale Wirtschaftsförderung im Umbruch Kundenmanagement in Bestandsentwicklung und im Standortmarketing praxisorientiert umsetzen. In: Standort - Zeitschrift für Angewandte Geographie (2003) 4, S. 161-166.

Porter, M. E. (1998): Clusters and the New Economics of Competition. In: Edward, Elgar (Eds.): Systems of Innovation: Growth, Competitiveness and Employment. MPG Books Ltd., Cornwall 2000, S. 309-322.

Porter, M. E. (2000): Locations, Clusters, and Company Strategy. In: Clark, G. L.; Feldman, M. P.; Gertler, M. S. (Eds.): Oxford Handbook of Economic Geography. Oxford University Press, New York, S. 253-274.

Reichling, T., Veith M. (2005): Expertise Sharing in a Heterogeneous Organizational Environment. In: Proc. of the 9th ECSCW. Springer, Dordrecht 2005, S. 325-345.

Reichling, T, Veith, M.. Wulf, V. (2007): Expert Recommender: Designing for a Network Organization, in: Computer Supported Cooperative Work: The Journal of Collaborative Computing (JCSCW), Vol. 16, No. 4- 5, 2007

Resnick, P., Varian, H. R. (1997): Recommender systems. Communications of the ACM, special issue, vol. 40, 1997, 56-58

Schiele, H. (Hrsg.) (2003): Der Standort-Faktor. Wie Unternehmen durch regionale Cluster ihre Produktivität und Innovationskraft steigern. Wiley-VCH Verlag, Weinheim 2003.

Sölvell, Ö., Lindqvist, G., Ketels, C. (2003): The Cluster Initiative Greenbook. Bromma tryck AB, Stockholm 2003.

Susman, G., Evered, R. (1978): An assessment of the scientific merits of action research, Administrative Science Quarterly, 23, 582-603

Wolfe, D. A. (2002): Social Capital and Cluster Development in Learning Regions. In: Holbrook, J. Adam; Wolfe, David Alexander (Eds.): Knowledge, Clusters and Learning Regions. Queen's University, Kingston. 
Wulf, V., Rohde, M. (1995): Towards an integrated organization and technology development, in Proc. of the conference on Designing interactive systems: processes, practices, methods, \& techniques, 1995, ACM Press, New York, pp. 55-64. 